\title{
The Temperature Dependence of Predation Stress and Prey Nutritional Stoichiometry
}

\author{
Oswald J. Schmitz * and Adam E. Rosenblatt \\ School of Forestry and Environmental Studies, Yale University, New Haven, CT, United States
}

Prey at risk of predation may experience stress and respond physiologically by altering their metabolic rates. Theory predicts that such physiological changes should alter prey nutrient demands from $\mathrm{N}$-rich to $\mathrm{C}$-rich macronutrients and shift the balance between maintenance and growth/reproduction. Theory further suggests that for ectotherms temperature stands to exacerbate this stress. But the behavior of many prey species facing perceived predation risk is the opposite of these predictions, consuming more $\mathrm{N}$-rich resources. Here we revisit the original Threshold Elemental Ratio (TER) theoretical framework that inspired the idea of shifts in elemental (C:N) stoichiometry in response to chronic predation stress to reconcile the different prey responses. We examine the interactive effects of predation stress and temperature stress by exploring mathematically how the component physiological variables that determine TER vary individually with temperature. These functional relationships are then embedded into the equation for TER to predict how $\mathrm{C}$ and $\mathrm{N}$ intake should vary with and without predation stress across

Edited by:

Gyöngyvér Mara, Sapientia Hungarian University of Transylvania, Romania

Reviewed by:

Jofre Carnicer, University of Groningen, Netherlands Ann Valerie Hedrick,

University of California, Davis, United States

*Correspondence:

Oswald J. Schmitz oswald.schmitz@yale.edu

Specialty section:

This article was submitted to Behavioral and Evolutionary Ecology, a section of the journal

Frontiers in Ecology and Evolution

Received: 31 January 2017 Accepted: 21 June 2017 Published: 14 July 2017

Citation: Schmitz OJ and Rosenblatt AE (2017)

The Temperature Dependence of Predation Stress and Prey Nutritional Stoichiometry. Front. Ecol. Evol. 5:73. doi: 10.3389/fevo.2017.00073 temperature gradients. This new theory reconciles the different prey responses and explains why and when species ought to consume more $\mathrm{N}$ vs. more $\mathrm{C}$ when stressed by perceived predation, depending on the nature of their thermal performance with rising temperature. The theory also points to new ways to conduct experimental evaluations testing the temperature sensitivity of prey to predation stress.

Keywords: foraging-predation tradeoff, metabolism, predation stress, stoichiometry, thermal performance

\section{INTRODUCTION}

The classic concept of adaptive foraging-predation risk trade-off has served as a straightforward generalization to enhance understanding of how species interactions shape community structure (Werner and Peacor, 2003; Preisser et al., 2005; Peckarsky et al., 2008) and ecosystem processes (Schmitz et al., 2008; Schmitz, 2010; Trussell and Schmitz, 2012). In this view, organisms maximize fitness by allocating less time to foraging and more time to vigilance in response to perceived predation risk. Increasing vigilance can involve prey simply reducing foraging time within a habitat, or more complex shifts between foraging habitats and refuge habitats. Such processes, along with others driven by predator-induction of prey defense morphology, have become collectively known as non-consumptive effects of perceived predation (Abrams, 2007).

Recent research has now revealed another kind of non-consumptive effect of perceived predation risk. It involves stress responses that, when chronic, can lead to persistent physiological changes like elevated metabolic rate (Beckerman et al., 2007; Slos and Stoks, 2008; Hawlena and Schmitz, 2010a; Zanette et al., 2011; Thaler et al., 2012; Clinchy et al., 2013; Van Dievel et al., 2016). This is an adaptive coping mechanism (Hawlena and Schmitz, 2010a). Elevated metabolic 
rate helps keep prey in a heightened state of alertness to increase their escape performance, thereby lowering the likelihood that predators will capture them (Hawlena et al., 2011; Zanette et al., 2011; Clinchy et al., 2013). But elevated metabolism arising from perceived predation risk may change organismal nutrient demand and hence the kinds of resources consumed by prey (McPeek et al., 2001; Hawlena and Schmitz, 2010b; Thaler et al., 2012). This also has implications for organismal fitness, but through a physiological trade-off in nutrient allocation between maintenance, growth, and reproduction.

Theory predicts that whenever mass-specific metabolic rate of prey exposed to chronic predation risk increases, prey should generally shift their nutrient intake and consume more carbohydrate than non-stressed prey (Hawlena and Schmitz, 2010a). This prediction derives from the idea that stressed animals should switch their life history strategies from one devoted to protein demanding growth or reproduction (production) to one of fulfilling energy demanding maintenance functions to cope with the added stress (Hawlena and Schmitz, 2010a). These shifts in nutrient intake should also be reflected in body element stoichiometry, with stressed prey having higher carbon $[\mathrm{C}]$ :nitrogen $[\mathrm{N}]$ ratios than non-stressed prey (Hawlena and Schmitz, 2010a). Some experiments have shown that such shifts in nutrient intake and body stoichiometry can indeed occur between conditions with and without perceived predation risk (McPeek et al., 2001; Hawlena and Schmitz, 2010b; Jansens et al., 2015). Other experiments have, however, shown that the predicted responses do not occur (Costello and Michel, 2013; Dalton and Flecker, 2014; Kaplan et al., 2014; Guariento et al., 2015; Kirschman et al., 2016; Van Dievel et al., 2016; Zhang et al., 2016), thereby calling into question the generality of the predicted stress response.

Here we revisit the original stoichiometric framework that inspired the idea of shifts in nutrient intake and body stoichiometry in response to chronic predation stress. We examine how predictions could change when other environmental conditions may cause additional stress, or when environmental conditions may trigger other kinds of adaptive responses by prey that could mitigate the stress response to perceived predation risk. This reconsideration is motivated by a recent experimental study (Schmitz et al., 2016) that showed the physiological response of ectothermic grasshopper (Melanoplus femurrubrum) prey to perceived predation risk can interact with environmental temperature. Grasshoppers reared at cooler temperatures $\left(15^{\circ} \mathrm{C}\right)$ grew little and developed slowly, survived poorly, and did not respond to perceived predation risk through diet shifts, preferring high dietary energy (carbohydrate-C) intake in risk and risk-free treatments simply to survive. Grasshoppers reared at intermediate temperature $\left(25^{\circ} \mathrm{C}\right)$ selected higher dietary protein- $\mathrm{N}$ and thus exhibited increased growth and development relative to those in cooler conditions. But they also responded to perceived predation risk, where individuals reared under perceived predation risk selected higher dietary carbohydrate- $\mathrm{C}$ than individuals reared in risk-free conditions. Individuals reared at the highest temperature $\left(35^{\circ} \mathrm{C}\right)$ grew large and developed rapidly, survived well, and did not respond to perceived predation risk through diet shifts, preferring high dietary protein- $\mathrm{N}$ intake in both risk and risk-free conditions. The study suggests that coping with predation stress was prioritized less than coping with temperature stress when individuals were living in a cold environment. Coping with predation stress was downplayed by individuals living in a hot environment because all individuals could rapidly develop through life cycle stages such that they became quickly invulnerable to predation. It was only at the intermediate temperature where grasshoppers exhibited the foraging-predation risk trade-off anticipated by theory (Hawlena and Schmitz, 2010a).

Our aim here is to begin reconciling why, when, and how the exceptions to the predictions have arisen. This is done in light of growing empirical insights from analyses of stress responses of prey facing perceived predation risk and additional insights form organismal thermal physiology. These insights will be synthesized using the original stoichiometric framework used to make predictions of prey stress responses to perceived predation risk. The framework helps guide the synthesis by specifying salient variables that need to be considered. This can lead to new predictions of how changing environmental temperature and predation risk, both singly and in combination, influence organismal elemental stoichiometry.

\section{BACKGROUND CONCEPTUAL THEORY}

Ecological stoichiometry aims to understand the flow of nutrients through food webs and ecosystems. Organisms are the building blocks of this framework because they mediate the flux of nutrients into their own bodies and the release of nutrients back into the environment to be recycled. Nutrient uptake by organisms is constrained by the demand for essential chemical elements (e.g., C, N, P) to fulfill physiological needs for growth, development, and reproduction, relative to elements available in macronutrients in food (Sterner and Elser, 2002). Limitation of the basic life processes of consumers by various elements will depend on their metabolic and somatic demands (Sterner and Elser, 2002). For example, stoichiometric models assume that $\mathrm{C}$ is used primarily to fuel respiration with some allocation to production, whereas $\mathrm{N}$ (or phosphorus $[\mathrm{P}]$ ) is used exclusively for production. Stoichiometric theory suggests that the extent to which elements are allocated to production arises from differences in both resource quality (C:N:P content) and quantity among environments. These factors may also determine which of the elements becomes most limiting to production. For example, food quality may be immaterial in environments with very low food quantity because most, if not all, resource intake will be devoted to supplying $\mathrm{C}$ for respiration with negligible amounts left over for production (Sterner, 1997). Consequently, there will be low demand for $\mathrm{N}$ or $\mathrm{P}$. Higher resource quantity allows excess intake to be allocated to production, at which time balancing elemental ratios factors into resource intake. Changes in organismal metabolic rate, and hence changes in resource demand, can likewise make a given quantity of food relatively more or less abundant, which could lead to similar shifts in $\mathrm{C}$ vs. $\mathrm{N}$ and $\mathrm{P}$ consumption. 
The mechanism determining this plasticity is encapsulated by the concept of the threshold elemental ratio (TER) - the dietary mixture where growth limitation switches from one element to another (Sterner, 1997; Frost et al., 2006; Cross et al., 2015). TER for $\mathrm{C}$ and other elements $\mathrm{X}$ (e.g., $\mathrm{N}$ or $\mathrm{P}$ ) reflects both animal body $\mathrm{C}: \mathrm{X}$ ratios and the proportion of ingested $\mathrm{C}$ used for growth (i.e., gross growth efficiency of $\left.\mathrm{C}, \mathrm{GGE}_{C}\right) . \mathrm{GGE}_{C}$ can be expressed as the percentage of ingested $C$ that was assimilated into new growth (Frost et al., 2006; Cross et al., 2015):

$$
G G E_{C}=\frac{\left(I_{C} \cdot A_{C}\right)-R_{C}}{I_{C}}
$$

where $A_{C}$ is the assimilation efficiency of $C, I_{C}$ is the mass-specific ingestion rate above a saturating food level, and $R_{C}$ is the massspecific respiration rate. The $T E R_{C: X}$ can be expressed as the product of physiological nutrient efficiencies and body elemental composition:

$$
\operatorname{TER}_{C: X}=\frac{A_{X}}{G G E_{C}} \cdot \frac{Q_{C}}{Q_{X}}
$$

where $A_{X}$ is the assimilation efficiency of element $X$, and $Q_{C}$ and $Q_{X}$ are the proportion of animal dry mass in $C$ and $X$. TER $C: X$ describes the degree to which energy $(C)$ and nutrients (X) are demanded physiologically to meet maintenance and growth requirements of organisms (Cross et al., 2015). A high C:X ratio indicates greater demand for (and hence limitation by) energyC; a low ratio indicates greater demand for (and limitation by) nutrients.

The original idea of stress responses induced by predation risk held that stress elevates $\mathrm{R}_{C}$ (Hawlena and Schmitz, 2010a) thereby decreasing $\mathrm{GGE}_{\mathrm{C}}$ which in turn increases $\mathrm{TER}_{C: X}$ (Hawlena and Schmitz, 2010a). The predicted consequence is that prey should shift nutrient demand from biomolecules such as $\mathrm{N}$-rich proteins that support growth and reproduction toward carbohydrates or fats (C-rich, N-poor) that fuel the heightened respiratory demands (Hawlena and Schmitz, 2010a). It has been hypothesized that for ectotherms, increasing temperature and thereby increasing $\mathrm{R}_{C}$, should exacerbate the effects of predation stress (Schmitz, 2013). This may indeed happen (Culler et al., 2014; Schmitz et al., 2016), but it is not universally true: the idea that temperature effects on organismal stoichiometry should conflate effects of stress from predation is based on the implicit assumption that all variables other than $R_{C}$ that determine the magnitude of $G G E_{C}$ and $T E R_{C: X}$ remain constant in the face of perceived predation risk and changes in other environmental conditions. Recent experiments evaluating the interplay between temperature and predation stress have shown that this assumption may not hold (Culler et al., 2014; Schmitz et al., 2016). It may also not hold for temperature effects on organismal stoichiometry more broadly (Cross et al., 2015). We therefore follow recommendations (Cross et al., 2015) to explore the stoichiometric consequences of changing conditions by systematically examining temperature effects on each of the variables determining $T E R_{C: X}$. We also examine how perceived predation risk can confound or conflate those responses depending on responses of prey.

\section{Temperature Effects on Variables}

Temperature effects on ectotherm fitness, mediated through physiology, can generally be described by a thermal performance curve that rises, peaks, and then declines across a gradient of increasing temperature (Chown and Nicholson, 2004; Angilletta, 2009; Clissold and Simpson, 2015). The thermal performance curve reflects the range of temperatures (thermal window: Pörtner and Farrell, 2008) that organisms can tolerate and still survive and reproduce. Organisms have likely evolved to operate in thermal windows that are as narrow as possible to minimize maintenance costs (Pörtner and Farrell, 2008). Thermal performance is routinely measured in terms of metabolic rate-temperature relationships (e.g., Figure 1). But it can also be characterized in terms of other performance measures related to fitness such as locomotor behavior, growth rate, and offspring production (Kingsolver and Woods, 1997; Chown and Nicholson, 2004; Clissold and Simpson, 2015). While the exact temperature range of the thermal window will vary by species (Pörtner and Farrell, 2008), as well as the local thermal conditions faced by geographically separated populations of a species (e.g., Rosenblatt et al., 2016), the general, qualitative features of the performance curve nonetheless can help conceptualize the interplay between temperature and predation stress. We do this here by characterizing patterns of thermal performance in each of the variables used to estimate $\mathrm{TER}_{C: X}$, using empirical insights from studies that have begun to explore the stoichiometric consequences of predation stress and temperature on ectotherm organismal performance.

A fundamental physiologic property (Chown and Nicholson, $2004)$ is that mass specific metabolic rate $\left(R_{C}\right)$ of ectotherms will rise, peak and then decline with increasing temperature (Figure 1). Experimental research has shown that predation stress can exacerbate $\mathrm{R}_{C}$ across the temperature gradient (Culler et al., 2014; Schmitz et al., 2016). Mass specific ingestion rate may follow a similar nonlinear trend across a temperature range (Kingsolver and Woods, 1997, 1998; Chown and Nicholson, 2004), whereas assimilation efficiency $\mathrm{A}_{C}$ might be approximated as a linearly increasing function of temperature (Culler et al., 2014; Clissold and Simpson, 2015; Schmitz et al., 2016). These empirical trends can be used to begin exploring how $\mathrm{GGE}_{C}$ and $\mathrm{TER}_{C: X}$ might vary with temperature. We do this here by approximating the empirical relationships of the component variables with temperature $(\mathrm{T})$, and substitute those functions

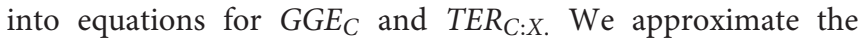
temperature dependent functions as:

$$
\begin{aligned}
I_{C} & =a_{I c}+b_{I c} T-c_{I c} T^{2} \\
R_{C} & =a_{R c}+b_{R c} T-c_{R c} T^{2} \\
A_{C} & =a_{A c}+b_{A c} T
\end{aligned}
$$

Substituting these functions into the equation for $\mathrm{GGE}_{C}$ and algebraically simplifying the coefficients gives:

$$
G G E_{C}=\frac{\hat{a}+\hat{b} T+\hat{c} T^{2}-\hat{d} T^{3}}{a_{I c}+b_{I c} T-c_{I c} T^{2}}
$$



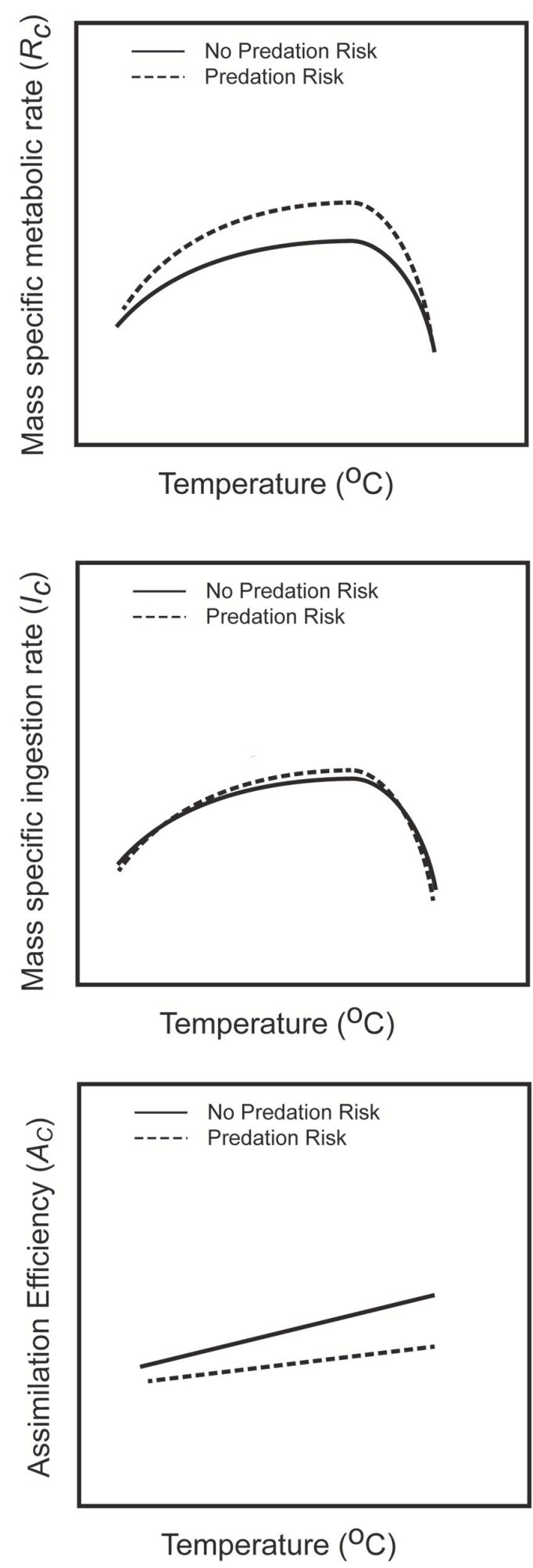

FIGURE 1 | Qualitative functional relationships depicting the thermal performance of prey metabolism, prey resource ingestion rate and assimilation efficiency in the presence and absence of perceived predation risk. These relations summarize known empirical relationships derived from experimental analyses reported in the literature. These relationships are characterized mathematically to predict the temperature sensitivity of prey selection for $\mathrm{C}$ and $\mathrm{N}$ in risk and risk free conditions.
Qualitatively, Equation (4) generally gives rise to a highly nonlinear function that rises, peaks and declines with rising temperature (Figure 2). It can be used to explore how perceived predation risk might influence the temperature dependent relationship by examining how the model parameters might change. It has been shown empirically that assimilation efficiency is lower when prey face perceived predation risk (Risk prey) than when facing no risk (No Risk prey), and efficiency for Risk prey rises more slowly with rising temperature than for No Risk prey (Schmitz et al., 2016). Hence, the magnitude of $a_{A c}$ and $b_{A c}$ in Equation (3c) will be lower for Risk prey than for No Risk prey. Across a temperature gradient, mass specific metabolic rate of Risk prey tends to be higher than for No Risk prey (Culler et al., 2014; Schmitz et al., 2016). Hence, as a first approximation one may assume that the magnitudes of $a_{R c}$ and $b_{R c}$ in Equation (3b) will be larger for Risk prey relative to No Risk prey. These assumptions imply further that in Equation (4), $\hat{a}$ and $\hat{b}$ for Risk prey will be less than for No Risk prey. Collectively, these parameter changes will lead to the same qualitative form of the temperature dependency of $G G E_{C}$ (Figure 2A). However, the curve describing $G G E_{C}$ for Risk prey is offset from the curve for No Risk prey.

The effects of temperature and perceived predation risk on $T E R_{C: X}$ can be explored by substituting the temperature dependent functional form of $G G E_{C}$ into Equation (2) and then comparing trends between Risk and No Risk conditions. Doing this, however, requires making assumptions about the way assimilation efficiency of nutrient $\mathrm{X}, A_{X}$, might vary with temperature, for which there is currently very limited general understanding (Cross et al., 2015), let alone understanding of how $A_{X}$ varies between Risk and No Risk conditions. We therefore consider several possible alternatives, beginning with the assumption that $A_{X}$ could remain constant, increase linearly, decrease linearly, or vary nonlinearly with rising temperature, based on some empirical insights (Culler et al., 2014; Dalton and Flecker, 2014; Lemoine and Shantz, 2016; Schmitz et al., 2016).

These assumptions give rise to at least four contingencies in which: (1) $A_{X}$ is constant for both No Risk and Risk prey across the temperature gradient, but with $A_{X}$ for Risk $<A_{X}$ for No Risk prey (Figure 2B); (2) $A_{X}$ is constant for No Risk prey and increases linearly with temperature for Risk prey (Figure 2C); $A_{X}$ is constant for No Risk prey and decreases linearly with temperature for Risk prey (Figure 2D); and $A_{X}$ for both No Risk and Risk prey changes nonlinearly with temperature in the same qualitative way as does $R_{C}$ or $I_{C}$ (Figure 1).

The contingencies show first that rising temperature should not always lead to increased intake of $C$ by prey stressed from perceived predation risk. In some cases (Figure 2B) C:X of Risk prey will always be lower than for No Risk prey, implying that Risk prey should always consume more $\mathrm{N}$ than No Risk prey. In other cases, rising temperature will cause C:X of No Risk prey and Risk prey to switch. For instance, C:X ratios of foods consumed by Risk prey may be higher than for No Risk prey up to a threshold temperature, and then switch to be lower. This means that Risk prey should consume more $\mathrm{C}$ with rising temperature up to a threshold, after which they are expected to consume more $\mathrm{N}$ than No Risk prey. The reverse may also happen (Figure 2D). Finally, the trend could be highly nonlinear 


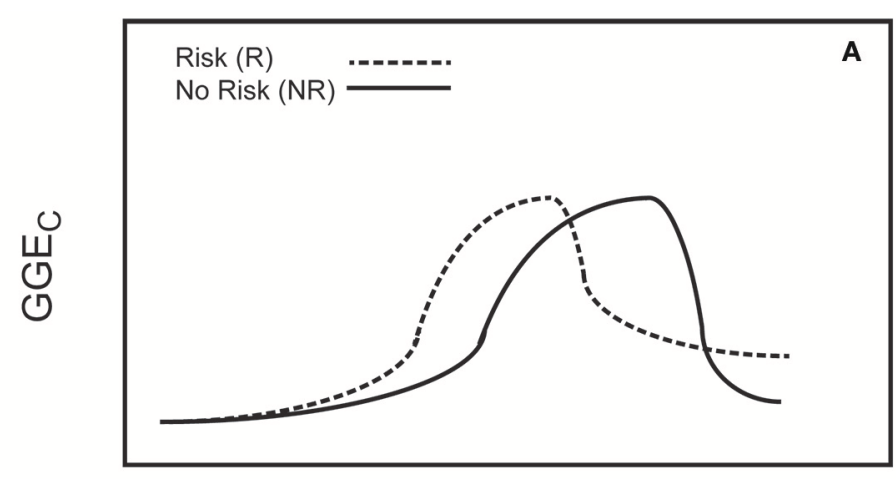

Temperature $\left({ }^{\circ} \mathrm{C}\right)$
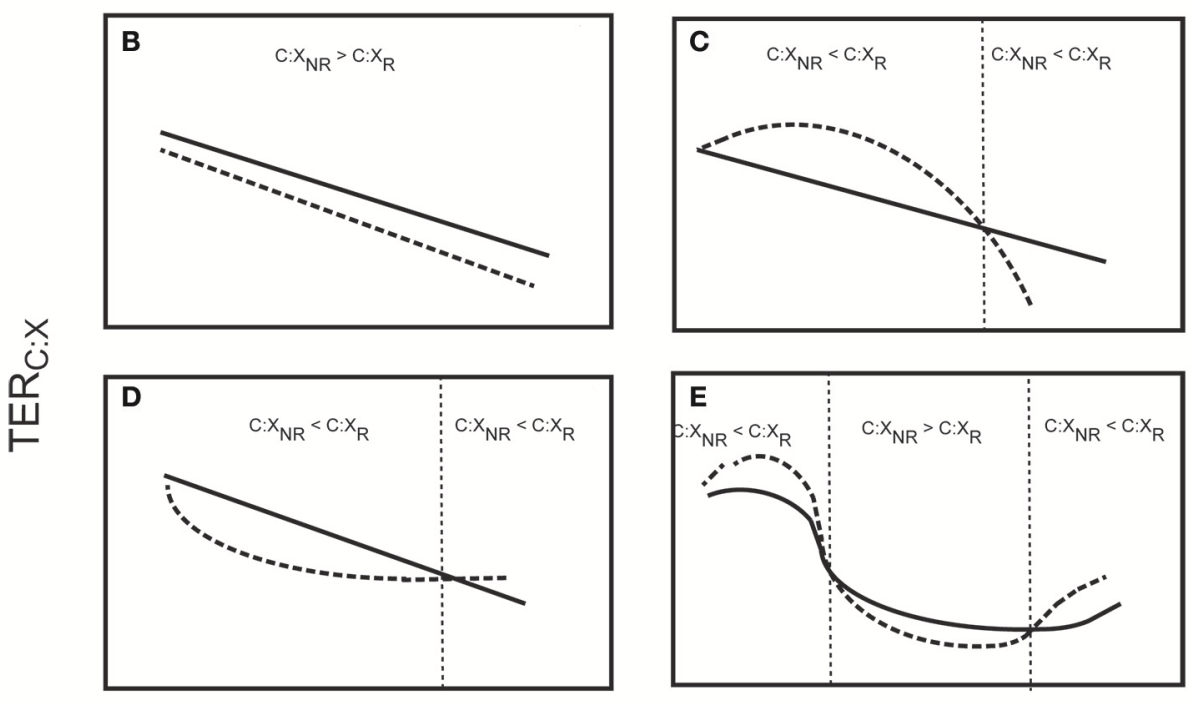

Temperature $\left({ }^{\circ} \mathrm{C}\right)$

FIGURE 2 | The temperature sensitivity of prey to perceived predation stress and implications for intake of dietary $\mathrm{C}$ and $\mathrm{N}$ based on functional relationships depicted in Figure 1. (A) Gross growth efficiency $\left(G G E_{C}\right)$ varies nonlinearly with temperature, but perceived predation risk offsets the curve relative to no-risk conditions. (B-E) The temperature sensitivity of the threshold elemental ratio TER, determined by the ratio of assimilation efficiency of $N / G G E_{C}$, is contingent on assumptions about how assimilation efficiency $A_{X}$ varies with temperature. The depictions assume that $A_{X}$ is constant for both risk and risk free conditions (B), is constant for risk free conditions but increases linearly with temperature for risk conditions (C), is constant for risk free conditions but decreases linearly with temperature for risk conditions (D), and changes nonlinearly (increasing and then decreasing) across a temperature gradient for both risk and risk free conditions (E). The figure shows temperature thresholds (vertical dotted lines) where prey facing risk would consume more $N$ than no risk prey $\left(C: X_{N R}>C: X_{R}\right)$, and when prey facing risk should consume more $\mathrm{C}$ than no risk prey $\left(C: X_{N R}<C: X_{R}\right)$. The figure illustrates that consumption ratios are highly dependent on the temperature conditions in which prey risk responses are evaluated.

across a temperature gradient leading to multiple switches where Risk prey may consume more $\mathrm{C}$ than No Risk prey, then more $\mathrm{N}$, then again more $\mathrm{C}$ (Figure 2E). The contingencies depicted in Figure 2 are not an exhaustive representation of possibilities. Rather their presentation is intended to reveal the range of different nonlinearities that could emerge, depending on the temperature dependency of the component variables. This thereby reinforces earlier arguments (Cross et al., 2015) for the need to better quantify how the model variables $R_{C}, I_{C}, A_{C}$, and $A_{X}$ all vary with temperature, let alone how they change with stress from perceived predation risk.

\section{Implications for Studies on the Stoichiometry of Predation Stress}

This new insight has several implications for conclusions drawn from studies testing effects of predation stress on ectotherm nutrient intake and physiological performance. First, the original argument that environmental warming should exacerbate the effects of stress from predation risk, causing prey to increase $\mathrm{C}$ consumption (Schmitz, 2013), is a special case of a broader range of outcomes. Second, the empirical finding that across a gradient of increasing temperature, Risk prey and No Risk prey consume equal amounts of $\mathrm{C}$ and $\mathrm{N}$, then deviate from each other with 
Risk prey consuming more $\mathrm{C}$, then switching where Risk prey consume equal or less $C$ than No Risk prey (Schmitz et al., 2016) could be explained by one of the contingencies (Figure 2C). Hence, the experimental outcome could be consistent with, but a generalization of, the original idea of the effects of predation stress on organismal stoichiometry (Hawlena and Schmitz, 2010a). Third, our results may also help offer clues as to why results of studies that tested for the effects of predation stress on nutrient intake by ectothermic species were counter to original predictions (Thaler et al., 2012; Costello and Michel, 2013; Dalton and Flecker, 2014; Kaplan et al., 2014; Guariento et al., 2015; Kirschman et al., 2016; Van Dievel et al., 2016).

The higher $\mathrm{N}$ intake by Risk prey than by No Risk prey observed in these studies has been attributed to developmental needs that facilitate prey escape from predation. Increased $\mathrm{N}$ intake under perceived predation risk is needed by aquatic prey like damselflies, fish, and amphibians to increase the development rate of locomotor traits that enable prey to escape their actively hunting predators, or for terrestrial caterpillars to grow rapidly to become too large for predators to dispatch them. Our analysis shows that this could counterintuitively be achieved whenever Risk prey lower their assimilation efficiency of N, relative to No Risk prey (Figure 2B). But studies have shown that assimilation efficiency may increase (Thaler et al., 2012; Dalton and Flecker, 2014), which should, according to our analysis, lead to higher $\mathrm{C}$ intake by Risk prey than No Risk prey (e.g., Figure 2C). Our analysis suggests that to maintain a higher net intake of $\mathrm{N}$, Risk prey that increase $\mathrm{N}$ assimilation efficiency would need to compensate by lowering their consumption rate of food $I_{C}$. This has indeed been observed experimentally (Thaler et al., 2012; Dalton and Flecker, 2014).

Several studies experimentally examining the interplay between temperature and predation risk have provided mixed support for the original idea that rising temperature should exacerbate predation stress causing prey to consume more $\mathrm{C}$ (Jansens et al., 2015; Kirschman et al., 2016; Van Dievel et al., 2016; Zhang et al., 2016). These studies used $2 \times 2$ factorial designs that crossed a temperature treatment with a perceived predation risk treatment. Our analysis reveals that mixed outcomes are to be expected with such experimental designs because the outcomes depend on the specific temperatures used in the experiments. A simple answer is that the experiments reared prey on both sides of a temperature threshold, in which case Risk prey on the warm side of the threshold would consume less $\mathrm{C}$ than individuals in cooler temperatures (e.g., Figure 2D, or Figure 2E left threshold). It could also arise if assimilation efficiency of $\mathrm{N}$ decreases with rising temperature (Figure 2D temperature range below the threshold) or if $\mathrm{N}$ assimilation increases with temperature, but the experimental temperatures put Risk prey on the descending side of the $T E R_{C: X}$ vs. temperature curve (Figure 2C). Essentially, the analysis shows that factorial experiments that merely cross one experimental temperature with perceived predation risk could give equivocal insight in the absence of knowing how each of the component model variables $\left(R_{C}, I_{C}, A_{C}\right.$, and $\left.A_{X}\right)$ vary with temperature and perceived predation risk.

\section{Moving Forward}

There is growing interest in examining the interactive effects of climate and other environmental stressors on organismal stoichiometry (Cross et al., 2015; Rosenblatt and Schmitz, 2016). Such examinations of the interactive effects of temperature and perceived predation risk on prey performance is in line with the growing need to understand how environmental warming will influence trophic interactions in communities. The current, small body of research examining effects on prey of interactions between temperature and perceived predation stress means that our current understanding is rather piecemeal, with limited ability to make empirical generalizations at this time.

Nevertheless, the field is also at an opportune juncture to improve how future studies are undertaken. Specifically, the existing research has revealed that prey responses to temperature and perceived predation risk are highly context dependent. It currently remains unclear whether or not the nature of nutrient intake in the presence and absence of perceived predation risk by different species is a unique attribute of each species or if they are temperature-specific manifestations of a more general temperature dependent response to predation stress (Schmitz et al., 2016). Our analysis presented here suggests the general TER framework of ecological stoichiometry can help to reconcile these different outcomes and suggests that they are part of a more general temperature dependent response to predation stress. However, our general framework also shows that outcomes will be contingent on how each of the component variables of the TER framework vary with temperature and predation stress. This highlights that experiments that simply manipulate temperature and perceived predation risk and measure prey consumption of $\mathrm{C}$ and nutrients (e.g., $\mathrm{N}$ or $\mathrm{P}$ ) could provide equivocal results if the treatment effects of the key underlying physiological variables $R_{C}$, $I_{C}, A_{C}$, and $A_{X}$ are not measured. Moreover, given the potential for threshold effects of increasing temperature identified in our qualitative analysis (Figure 2), we suggest that studies examine the interplay between temperature and perceived predation risk across temperature gradients.

Such studies need to be predicated on knowing the thermal tolerance range of the focal species (i.e., the range between the minimum and maximum thermal limits). Experimentation would then systematically increment temperature within this range as well as the presence and absence of perceived predation risk (e.g., Culler et al., 2014). The key physiological variables would then be measured at the different temperatures. Given the potential for nonlinear responses of prey to temperature and predation stress, we suggest measuring physiological variables across a gradient of at least four temperatures, although more will allow clearer characterization of the degree of nonlinearity (e.g., Culler et al., 2014). Such studies can provide insight into the nature of organismal performance curves (e.g., Culler et al., 2014; Schmitz et al., 2016) that can help inform which of the potential contingencies (Figures 2B-E) can be expected for a particular study organism.

There is also a need to explore the connection between $T E R_{C: X}$ and the biochemical compounds that make up nutrients. This is because animals do not metabolize elements, but instead 
metabolize biochemicals (Anderson et al., 2004; Wilder and Jeyasingh, 2016). TER $C: X$ is a first approximation that implicitly equates energy and nutrient selection with the total content of elemental $\mathrm{C}$ and $\mathrm{X}$ (e.g., $\mathrm{N}$ or $\mathrm{P}$ ) in food resources. Because elements are bound up in biochemicals such as carbohydrates, fats, and proteins, this assumes that $\mathrm{C}, \mathrm{N}$, and $\mathrm{P}$ are constant fractions of those biochemicals. But this assumption may not always hold, especially in terrestrial plants where elements contained in the digestible fraction of food may be uncorrelated with the fraction of total available elements (Anderson et al., 2004; Wilder and Jeyasingh, 2016). And, these digestible fractions can shift with changing environmental temperature (Zvereva and Kozlov, 2006; Rosenblatt and Schmitz, 2016; Wilder and Jeyasingh, 2016; Zhang et al., 2016). The conceptual theory also assumes that animals derive their energy- $\mathrm{C}$ from carbohydrates. But stressed prey may either consume more protein- $\mathrm{N}$ for energy or metabolize stored body protein to release energy via gluconeogenesis (Hawlena and Schmitz, 2010b) especially when other energy sources are at low concentrations. The effect of increased $\mathrm{N}$ intake for energy is not accounted for in estimation of $T E R_{C: X}$, which could then lead to incorrect predictions about dietary elemental intake of $\mathrm{C}$ and $\mathrm{N}$ under stress. Thus, a future avenue of research might consider how to revise $T E R_{C: X}$ theory to accommodate the need to consider diet selection for elements contained within biochemicals. In addition, exploration of how organisms select and utilize different biochemicals as a means to balance competing demands of allocating nutrients to cope with the stressors and allocating for growth and

\section{REFERENCES}

Abrams, P. A. (2007). Defining and measuring the impact of dynamic traits on interspecific interactions. Ecology 88, 2555-2562. doi: 10.1890/06-1381.1

Anderson, T. R., Boersma, M., and Raubenheimer, D. (2004). Stoichiometry: linking elements to biochemicals. Ecology 85, 1193-1202. doi: 10.1890/02-0252

Angilletta, M. J. (2009). Thermal Adaptation: A Theoretical and Empirical Synthesis. Oxford: Oxford University Press.

Beckerman, A. P., Wieski, K., and Baird, D. J. (2007). Behavioural versus physiological mediation of life history under predation risk. Oecologia 152, 335-343. doi: 10.1007/s00442-006-0642-6

Chown, S. L., and Nicholson, S. W. (2004). Insect Physiological Ecology. Oxford: Oxford University Press.

Clinchy, M., Sheriff, M. J., and Zanette, L. Y. (2013). Predator-induced stress and the ecology of fear. Funct. Ecol. 27, 56-65, doi: 10.1007/s00442-014-3084-6

Clissold, F. J., and Simpson, S. J. (2015). Temperature, food quality and life history traits of herbivorous insects. Curr. Opin. Insect Sci. 11, 63-70. doi: 10.1016/j.cois.2015.10.011

Costello, D. M., and Michel, M. J. (2013). Predator induced defenses in tadpoles confound body stoichiometry predictions of the general stress paradigm. Ecology 94, 2229-2236. doi: 10.1890/12-2251.1

Cross, W., Hood, J. M., Benstead, J. P., Huryn, A. D., and Nelson, D. (2015). Interactions between temperature and nutrients across levels of ecological organization. Global Change Biol. 21, 1025-1040. doi: 10.1111/gcb.12809

Culler, L. E., McPeek, M. A., and Ayers, M. P. (2014). Predation risk shapes thermal physiology of a predaceous damselfly. Oecologia 176, 653-660. doi: 10.1007/s00442-014-3058-8

Dalton, C. M., and Flecker, A. S. (2014). Metabolic stoichiometry and the ecology of fear in Trinidadian guppies: consequences for life-histories and stream ecosystems. Oceologia 176. 691-701. doi: 10.1007/s00442-014-3084-6 development would help inform such revisions (Schmitz et al., 2016).

In conclusion, the realization that non-consumptive effects of perceived predation can be important drivers of food web, community, and ecosystem dynamics was a crucial step forward in our understanding of ecological processes. However, rapid environmental shifts brought about by climate change and other global change drivers demand that we integrate the study of predation risk with our understanding of the ecological effects of other stressors. As part of this effort, we suggest that a fruitful avenue to pursue would be to build on existing linkages between ecological stoichiometry, nutrition, and phenotypic responses of prey to consider multiple environmental stressors (Rosenblatt and Schmitz, 2014, 2016). By expanding our understanding of the effects of predation risk on prey nutritional ecology across a range of potential future climates, our ability to accurately predict how future food webs and ecosystems may change will become more robust and reliable (Rosenblatt and Schmitz, 2016).

\section{AUTHOR CONTRIBUTIONS}

All authors listed have made a substantial, direct and intellectual contribution to the work, and approved it for publication.

\section{ACKNOWLEDGMENTS}

This research was support by US National Science Foundation grant DEB-1354762 to OS.
Frost, P. C., Benstead, J. P., Cross, W. F., Hillebrand, H., Larson, J. H., Xenopoulos, M. A., et al. (2006). Threshold elemental ratios of carbon and phosphorus in aquatic consumers. Ecol. Lett. 9, 774-779. doi: 10.1111/j.1461-0248.2006.00919.x

Guariento, R. D., Carniero, L. S., Jorge, J. S., Borges, A. N., Esteves, F. A., and Caliman, A. (2015). Interactive effects of predation risk and conspecific density on the nutrient stoichiometry of prey. Ecol. Evol. 5, 4747-4756. doi: 10.1007/s00442-014-3084-6

Hawlena, D., Hughes, K. M., and Schmitz, O. J. (2011). Trophic trait plasticity in response to changes in resource availability and predation risk. Funct. Ecol. 25, 1223-1231. doi: 10.1111/j.1365-2435.2011.01891.x

Hawlena, D., and Schmitz, O. J. (2010a). Herbivore physiological response to fear of predation and implications for ecosystem nutrient dynamics. Proc. Nat. Acad. Sci. U.S.A. 107, 15503-15507. doi: 10.1073/pnas.1009300107

Hawlena, D., and Schmitz, O. J. (2010b). Physiological stress as a fundamental mechanism linking predation to ecosystem processes. Am. Nat. 176, 537-556. doi: 10.1086/656495

Jansens, L., Van Dievel, M., and Stoks, R. (2015). Warming reinforces nonconsumptive predator effects on prey growth, physiology, and body stoichiometry. Ecology 96, 3270-3280. doi: 10.1890/15-0030.1

Kaplan, I., McArt, S. H., and Thaler, J. (2014). Plant defense and predation risk differentially shape patterns of consumption, growth and digestive efficiency in a guild of leaf-chewing insects. PLoS ONE 9:e93714. doi: 10.1371/journal.pone.0093714

Kingsolver, J. G., and Woods, H. A. (1997). Thermal sensitivity of growth and feeding in Manduca sexta caterpillars. Physiol. Zool. 70, 631-638. doi: $10.1086 / 515872$

Kingsolver, J. G., and Woods, H. A. (1998). Interactions of temperature and dietary protein concentration in growth and feeding of Manduca sexta caterpillars. Physiol. Entomol. 23, 354-359. doi: 10.1046/j.1365-3032.1998.00105.x 
Kirschman, L. J., Haslett, S., Fritz, K. A., Whiles, M. R., and Warne, R. W. (2016). Influence of physiological stress on nutrient stoichiometry in larval amphibians. Physiol. Biochem. Zool. 89, 313-321. doi: 10.1086/687047

Lemoine, N. P., and Shantz, A. A. (2016). Increased temperature causes protein limitation by reducing the efficiency of nitrogen digestion in the ectothermic herbivore Spodoptera exigua Physiol. Entomol. 122, 1669-1678. doi: 10.1111/phen.12138

McPeek, M. A., Grace, M., and Richardson, J. M. L. (2001). Physiological and behavioral responses to predators shape the growth/predation risk trade-off in damselflies. Ecology 82, 1535-1545. doi: 10.1890/00129658(2001)082[1535:PABRTP]2.0.CO;2

Peckarsky, B. L., Abrams, P. A., Bolnick, D. I., Dill, L. M., Grabowski, J. H., Luttbeg, B., et al. (2008). Revisiting the classics: considering nonconsumptive effects in textbook examples of predator-prey interactions. Ecology 89, 2416-2425. doi: 10.1890/07-1131.1

Pörtner, H. O., and Farrell, A. P. (2008). Physiology of climate change. Science 322, 690-692. doi: 10.1126/science.1163156

Preisser, E., Bolnick, D. I., and Benard, M. F. (2005). Scared to death? The effects of intimidation and consumption in predator-prey interactions. Ecology 86, 501-550. doi: 10.1890/04-0719

Rosenblatt, A. E., Crowley, B. T., and Schmitz, O. J. (2016). Linking trophic interactions to plasticity in thermal sensitivity of geographically separated populations of a herbivore. Evol. Ecol. 30, 649-661. doi: 10.1007/s10682016-9827-6

Rosenblatt, A. E., and Schmitz, O. J. (2014). Interactive effects of multiple climate change variables on trophic interactions: a meta-analysis. Climate Change Responses 1:8. doi: 10.1186/s40665-014-0008-y

Rosenblatt, A. E., and Schmitz, O. J. (2016). Climate change, nutrition, and bottom-up and top-down food web processes. Trends Ecol. Evol. 31, 965-975. doi: $10.1016 /$ j.tree.2016.09.009

Schmitz, O. J. (2010). Resolving Ecosystem Complexity. Princeton: Princeton University Press.

Schmitz, O. J. (2013). Global climate change and the evolutionary ecology of ecosystem function. Ann. N.Y. Acad. Sci. 1297, 61-72. doi: 10.1111/nyas.12181

Schmitz, O. J., Grabowski, J. H., Peckarsky, B. L., Preisser, E. L., Trussell, G. C., and Vonesh, J. R. (2008). From individuals to ecosystems: toward an integration of evolutionary and ecosystem ecology. Ecology 89, 2436-2445. doi: $10.1890 / 07-1030.1$

Schmitz, O. J., Rosenblatt, A. E., and Smylie, M. (2016). Temperature dependence of predation stress and the nutritional ecology of a generalist herbivore. Ecology 97, 3119-3130. doi: 10.1002/ecy.1524

Slos, S., and Stoks, R. (2008). Predation risk induces stress proteins and reduces antioxidant defense. Funct. Ecol. 22, 637-642. doi: 10.1111/j.1365-2435. 2008.01424.x

Sterner, R. W. (1997). Modelling interactions of food quality and quantity in homeostatic consumers. Freshwater Biol. 38, 473-481. doi: 10.1046/j.1365-2427.1997.00234.x
Sterner, R. W., and Elser, J. J. (2002). Ecological Stoichiometry: The Biology of Elements: From Molecules to the Biosphere. Princeton: Princeton University Press.

Thaler, J. S., McArt, S. H., and Kaplan, I. (2012). Compensatory mechanisms for ameliorating the fundamental trade-off between predator avoidance and foraging. Proc. Nat. Acad. Sci. U.S.A. 109, 12075-12080. doi: 10.1073/pnas.12080 70109

Trussell, G. C., and Schmitz, O. J. (2012). "Species functional traits, trophic control, and the ecosystem consequences of adaptive foraging in the middle of food chains," in Trait-Mediated Indirect Interactions: Ecological and Evolutionary Perspectives, eds T. Ohgushi, O. J. Schmitz, and R. D. Holt (Cambridge: Cambridge University Press), 324-338.

Van Dievel, M., Janssens, L., and Stoks, R. (2016). Short- and long-term behavioural, physiological and stoichiometric responses to predation risk indicate chronic stress and compensatory mechanisms. Oecologia 181, 347-357. doi: 10.1007/s00442-015-3440-1

Werner, E. E., and Peacor, S. D. (2003). A review of trait-mediated indirect interactions in ecological communities. Ecology 84, 1083-1100. doi: 10.1890/ 0012-9658(2003)084[1083:AROTII]2.0.CO;2

Wilder, S. M., and Jeyasingh, P. D. (2016). Merging elemental and macronutrient approaches for a comprehensive study of energy and nutrient flows. J. Anim. Ecol. 85, 1427-1430. doi: 10.1111/1365-2656. 12573

Zanette, L. Y., White, A. F., Allen, M. C., and Clinchy, M. (2011). Perceived predation risk reduces the number of offspring songbirds produce per year. Science 334, 1398-1401. doi: 10.1126/science. 1210908

Zhang, C., Jansen, M., De Meester, L., and Stoks, R. (2016). Energy storage and fecundity explain deviations from ecological stoichiometry predictions under global warming and size-selective predation. J. Anim. Ecol. 85, 1431-1441. doi: 10.1111/1365-2656.12531

Zvereva, E., and Kozlov, M. V. (2006). Consequences of simultaneous elevation of carbon dioxide and temperature for plant-herbivore interactions: a metaanalysis. Global Change Biol. 12, 27-41. doi: 10.1111/j.13652486.2005.01086.x

Conflict of Interest Statement: The authors declare that the research was conducted in the absence of any commercial or financial relationships that could be construed as a potential conflict of interest.

Copyright (๑) 2017 Schmitz and Rosenblatt. This is an open-access article distributed under the terms of the Creative Commons Attribution License (CC BY). The use, distribution or reproduction in other forums is permitted, provided the original author(s) or licensor are credited and that the original publication in this journal is cited, in accordance with accepted academic practice. No use, distribution or reproduction is permitted which does not comply with these terms. 